\title{
Quantum mechanics students' understanding of normalization
}

\author{
Kevin Lee Watson ${ }^{1}$
${ }^{1}$ Mathematics Department, Virginia Tech, 225 Stanger Street, Blacksburg, VA, 24061-0123
}

\begin{abstract}
Normalization is a particularly important concept within quantum mechanics due to the probabilistic nature of quantum systems. However, students' understanding of normalization has not been an explicit focus in past studies. In this paper, I will present a preliminary framework for students' understanding of mathematical norms and normalization of vectors, using interviews with quantum mechanics students to illustrate how the framework can be used to model and make sense of students' reasoning about the normalization of vectors.
\end{abstract}

\section{I.INTRODUCTION}

Normalization of vectors from various vector spaces (e.g., $\mathbb{R}^{n}, \mathbb{C}^{n}$, function spaces) and the resultant normalized vectors are mathematically important for a variety of contexts. Some examples include finding directional derivatives in multivariable calculus, the development of orthonormal bases through the Gram-Schmidt process in Linear Algebra and Numerical Analysis, and, more pertinently for undergraduate physics educators, creating mathematical models for states of quantum mechanical systems. Despite the applicability of normalization to a wide variety of concepts in science and mathematics, students' understanding of norms and normalization has not been widely studied. Studies that examined students' understanding of absolute value have come close to the topic of norms and normalization [1-2], but have not directly addressed them.

In this paper, I present a preliminary framework for students' understanding of mathematical norms and normalization of vectors, with two goals in mind. First, the framework could help us better understand student thinking and reasoning about vector normalization as well as give us greater insight into why students might struggle or excel in using these concepts. Second, the framework could identify particular aspects of norms and normalization students need to grasp in order to have a robust understanding of these concepts.

To this end, I first explain how the framework was theoretically and empirically developed. Next, I present the framework and explain the components and connections involved therein. I then use the framework to model and make sense of two students' problem solving processes as they work to normalize vectors from $\mathbb{R}^{2}$ and $\mathbb{C}^{2}$, followed by some conclusions and ideas for future research.

\section{II.DEVELOPMENT OF THE FRAMEWORK}

In many ways, the framework developed and used herein was inspired and influenced by Zandieh's [3] framework for student understanding of derivatives, and Lockwood's [4] model of students' combinatorial thinking. Similar to the work of Lockwood [4], I used a conceptual analysis [5] or "a detailed description of what is involved in knowing a particular (mathematical) concept" [6] to create this framework of students' understanding of norms and normalization. This conceptual analysis involved an iterative process of moving back and forth between my own theoretical thinking about the constructs involved in understanding norm and normalization, and student interview data. This interview data consists of hour-long, video-recorded, semi-structured interviews with multiple students at three different collection sites: (1) nine physics students from a university in the northwestern United States interviewed at the beginning of a junior-level quantum mechanics course; (2) nine physics students from a university in the northeastern United States interviewed at the beginning of a senior-level quantum mechanics course; and (3) two junior-level linear algebra students and two sophomore-level multivariable calculus students interviewed two-thirds into the semester from a university in the southeastern United States. Although the interviews from all three sites informed the framework development, in this paper I focus on the nine physics students from the first collection site, the data analysis of which consisted of: (1) watching the section of the interview in which students worked to normalize vectors from $\mathbb{R}^{2}$ and $\mathbb{C}^{2}$ and writing a summary of each student's thoughts about normalizing a vector; (2) coding the transcript of this normalization section for each student; and (3) examining how the framework could be used to model and make sense of each student's thinking and reasoning about norms and normalization, modifying the framework as necessary.

\section{III.FRAMEWORK FOR STUDENTS' UNDERSTANDING OF NORMALIZATION}

Figure 1 presents the framework in its entirety as a snapshot of the various components that are involved in understanding norms and normalization. It is meant to be an organizational tool for analyzing and thinking about students' understandings of these concepts. I contend that understanding normalization essentially involves three major components, namely: the norm of a vector, procedures for normalizing a vector, and what a normalized vector is (as conveyed by the three large ellipses in Fig. 1). I expand on the contents of these ellipses in the following subsections. The lack of directional arrows in the figure is deliberate, as any component could inform how a student thinks about any of the other components. Finally, students' understandings of norms and normalization do not necessarily include all of these components and connections; as such, when using the 
framework to model a student's understanding, components and connections presented in Fig. 1 could be missing for a particular student's model.

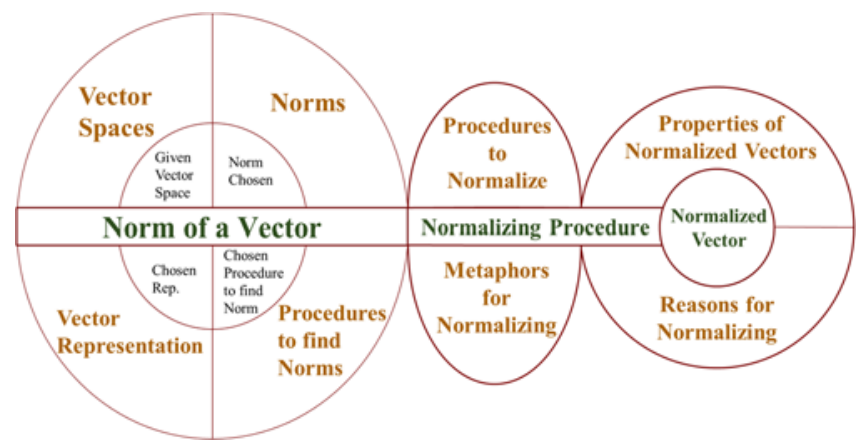

FIG 1. Framework for students' understanding of norms and normalization

\section{A. Norm of a vector}

When students find the norm of a specific vector, I have found four elements that can influence or determine how a student does this, namely: the vector space the given vector is an element of, the representation chosen for the vector, the chosen norm function to be used, and the procedure chosen for finding that norm (these four aspects are represented by the inner ellipse on the left of Figure 1). Furthermore, a student's broader understanding of vector spaces, representations of vectors, norms, and different procedures for finding norms can also inform and influence how the student finds the norm of a specific vector

A students' understanding of vector spaces could include examples of several vector spaces, such as $\mathbb{R}^{n}, \mathbb{C}^{n}$, or $L^{2}$ function space, although many students may only have experience with vectors in $\mathbb{R}^{n}$. Mathematically sophisticated students may also be able to draw on their understanding of the formal definition of vector space. Altogether these can influence how a student thinks about and understands a vector, which can further inform and influence their normalization of it.

A students' understanding of vector representations could include examples of several types: (1) algebraic notations (including using a letter with a special marking such as an arrow, bar, or hat; denoting a vector in $\mathbb{R}^{2}$ or $\mathbb{R}^{3}$ using a linear combination of the $\hat{\imath}, \hat{\jmath}$, and $\hat{k}$ unit vectors; using Dirac notation; or writing a function algebraically); (2) graphical notations (including graphs of functions, points on a Cartesian coordinate system, or directional arrows); and (3) matrix notation, in which vectors are represented as columns or rows. Each representation choice can affect how a student thinks about finding the norm of a vector. Furthermore, a student's understanding of why we use representations, and ability to select the best or most useful representation for a given task-which is part of Meta-Representational Competence [7-8] — could also impact a student's thinking about norms and normalization.
The only norm many undergraduate students are explicitly aware of knowing is the Euclidean Norm on $\mathbb{R}^{n}$, because most have only heard the term "norm" in conjunction with real vectors. However, mathematically sophisticated students may also know examples of other norms, or even the formal definition of norm. Any of these ideas can shape how a student approaches finding the norm of or normalizing a vector.

While it may seem strange to talk about procedures for finding norms apart from norms themselves, analyzing students' work revealed there is great variety in how students approach finding the norm of a vector. For instance, a few ways students can find the Euclidean norm of a vector in $\mathbb{R}^{2}$ are to take the square root of the sum of the squares of the components, take the square root of the dot product of the vector with itself, or graph the vector on a Cartesian plane and use the Pythagorean Theorem to find the length. While all of these are equivalent, each procedure can lead to different ways of thinking about finding the norm of a real vector. Additionally, when students normalize any vector, they can draw on their understanding of the connections between different equivalent procedures for finding the norm to inform their work.

\section{B. Normalizing procedure}

There are several different ways a student can normalize a vector. Some examples include: (1) dividing the vector by its norm, length, or magnitude; (2) multiplying the vector by the reciprocal of its norm; or (3) multiplying the vector by an unknown constant before finding its norm, setting it equal to one, and solving for the normalization constant. Moreover, there seem to be essentially two metaphorical expressions [9] students call upon when normalizing a vector which influence how they think about, and even notate, the normalized vector. The transformation/morphing metaphor views normalizing as a procedure that transforms or morphs the original vector into the normalized one. An example of using this metaphor is when a student talks about shrinking the original vector down to a length of one. The production metaphor views normalizing as a procedure that produces a vector that is in the "same direction" as the original vector, but has a length of one. Students who call upon this metaphor usually make a point of denoting the normalized vector differently from the original vector, such as adding a hat to the letter representing the original vector.

\section{Normalized Vector}

A student's understanding of normalized vectors includes ideas about properties of normalized vectors and reasons why normalization is important. The properties could include normalized vectors having a norm, length, or magnitude of one, and being in the same direction as the original vector. Understanding the reasons for normalization could include: the common idea that normalizing "gets rid of the magnitude", leaving you with direction only; probabilistic 
modeling (such as in quantum mechanics); or, looking at unit rates of change (such as with directional derivatives in multivariable calculus). Still, others could understand normalization as a rule or procedure that must be carried out simply because the book or teacher told them to do so.

\section{IV.EXAMPLES OF USING THE FRAMEWORK TO MODEL STUDENTS' UNDERSTANDING}

I now demonstrate how the framework can be used to model and make sense of students' understandings of norms and normalization. When using the framework to create a model, one can fill in the various components with details about the students' demonstrated understandings as they talk about and work on normalization problems, such as different vector spaces, representations, norms, and procedures they can draw upon. When students show no evidence of understanding particular components of the framework, this part of the framework is not included in the student's model. Lastly, examining students' attempts to normalize a specific vector (filling in the inner ellipse of Figure 1 accordingly), one can better understand why that student struggles or succeeds in normalizing that vector.

The two students focused on here, Danielle and Drake, are illustrative examples of how the elements and connections of the framework come together to inform each student's normalization of vectors. Both students were first asked to normalize $\left[\begin{array}{l}5 \\ 2\end{array}\right]$ and explain their work, and then asked to normalize $\left[\begin{array}{c}3+2 i \\ 4-i\end{array}\right]$, similarly explaining their thoughts and actions. It is noteworthy that this complex vector would not necessarily be common in a quantum mechanics class, as only the relative phase between components of a state vector is physically measurable, allowing the conventional choice of a real-valued first component; nevertheless, students' reasoning about this complex vector has been particularly insightful.

\section{A. Danielle}

When asked to normalize the real vector, Danielle immediately said this means to find the length or magnitude of the vector. In doing so, she was able to draw on multiple representations of vectors (including algebraic, geometric, and matrix representations), and mentioned how different procedures for finding norms could be equivalent:

Danielle: $\mathrm{OK}$, um, so, the norm of a vector is its length, and the - you can think of it as a triangle. Right? And then you can say, well, $\mathrm{v}$ - the length of $\mathrm{v}$ equals the square root of ... a squared plus b squared. Or, you could think of it as dotting a vector with itself, which is going to give you the square of the length. ... And, you get the same thing, you get the same idea. But, you get there slightly different ways.

While she was also able to explain how a norm could be found for a vector larger than two-dimensions, she seemed to only think about real vectors, and her demonstrated understanding of norm only included the Euclidean norm for real vectors. Furthermore, although able to explain and give examples of unit vectors, her understanding of normalization did not seem to be connected to ideas of creating a unit vector.

With this information, I created a model (see Fig. 2) of Danielle's understanding of normalization by only including the Norm of a Vector ellipse from the framework, making note that "normalize" and "norm of a vector" meant the same thing to her. This left out the "Normalizing Procedure" and "Normalized Vector" ellipses from the framework, as her understanding of normalization did not include these concepts. Additionally, a separate circle for her understanding of unit vectors was included to illustrate disconnection between her understanding of normalization and unit vectors.

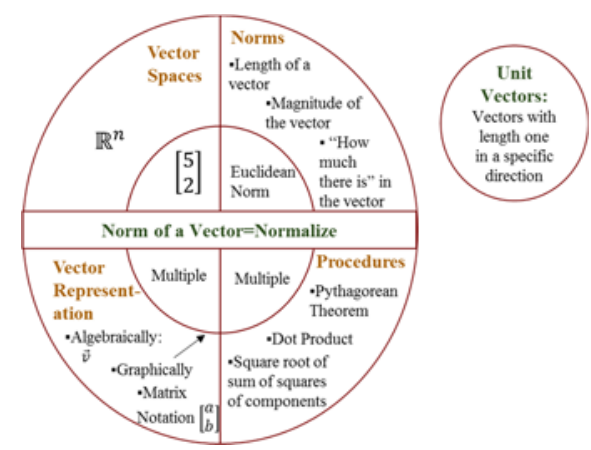

FIG 2. Model of Danielle's understanding: normalizing $\left[\begin{array}{l}\mathbf{5} \\ \mathbf{2}\end{array}\right]$

We can now make sense of Danielle's attempt to normalize the complex vector by imagining $\left[\begin{array}{l}\mathbf{5} \\ \mathbf{2}\end{array}\right]$ being replaced by $\left[\begin{array}{c}3+2 i \\ 4-i\end{array}\right]$ in the model above. Her limited understanding of norms and normalization became problematic at this point, seeing a necessity to throw out using the Pythagorean Theorem, explaining how there was not a way for her to think geometrically about this vector. She then decided to use the square root of the dot product to find the length of the vector (a procedure for finding the Euclidean norm of real vectors), though she was unsure of the result.

Danielle: So, the norm of w [inaudible] would be [writes $\sqrt{30+4 i}$ ]. I guess. [Chuckles].

Interviewer: Um, talk to me about why you're - why you added the "I guess."

Danielle: It looks weird. Like, you know, I, I just haven't, I haven't done it with complex numbers before, so my, sort of, intuition on whether or not it's right isn't there. So, assuming I haven't made a mistake in the math, if you dot two vectors with - 'kay, if you dot a vector with itself, it should give you the norm.

Ultimately her solution to finding the length or magnitude of the complex vector was incorrect; however, we can see her 
drawing on her understanding of normalization as she attempted to do so.

\section{B. Drake}

In contrast to Danielle, Drake described normalization as the process of dividing a vector by its magnitude to produce another vector in the same direction as the original vector, but with a length of one. In addition to having multiple vector representations and procedures for finding norms to draw upon in his understanding, Drake knew you could find the norm of a complex number, and understood that norms should always be real-valued. The model of Drake's understanding is presented in Fig. 3, and demonstrates a more complete understanding of norms and normalization than Danielle.

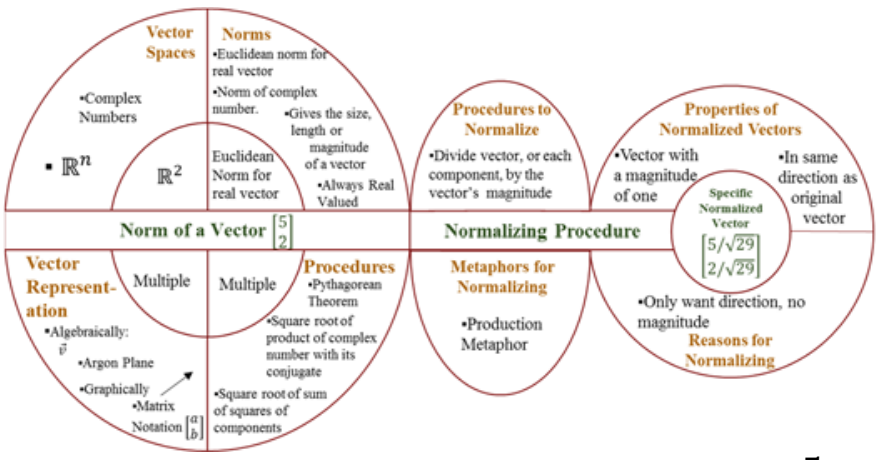

FIG 3. Model of Drake's understanding: normalizing $\left[\begin{array}{l}5 \\ 2\end{array}\right]$

When Drake was asked to normalize $\left[\begin{array}{c}3+2 i \\ 4-i\end{array}\right]$, he was able to draw on this broader understanding, especially the essential idea that norms should always be real valued, and found the norm by taking the square root of the dot product of the vector with a vector whose components were complex conjugates of the original vector:

Drake: Mmm, you have to use the complex conjugate 'cause you want to find, like, a real length. ... basically if you, if you did u dotted with u-star, and then squarerooted that, you would have the magnitude of, you'd have the magnitude of $u$.

He then correctly explained how to normalize the vector by dividing each component by this norm. Drake did, however, experience difficulties trying to visualize the complex vector, and was never satisfied by his attempts to graph the vector on the Argon plane. Moreover, Drake saw normalization as a way to "get rid of" a vector's magnitude to leave only the direction.

\section{V.CONCLUSION}

We can often think about normalizing vectors as simple, something that should not create much trouble for students. However, normalization can actually be quite intricate. Furthermore, the unfamiliar vector spaces in quantum mechanics, such as $\mathbb{C}^{n}$ to model Spins, can present unique challenges to students. In order to better prepare students to work with normalization in unfamiliar vector spaces, it is important that they are aware of important properties of norms (e.g., always real valued), as well as the existence of other norms for different vector spaces other than $\mathbb{R}^{n}$. Furthermore, instructors should explicitly help students understand the importance of and reason for normalizing vectors in specific contexts. Doing so might help students avoid thinking normalization just means to find the length of a vector (like Danielle), or naïvely thinking normalization "gets rid of" a vector's magnitude (like Drake).

In future research, I hope to explore students' understandings of normalizing wave functions, as well as examine ways in which instructors might help their students' better understand normalization and its importance in various STEM applications.

\section{ACKNOWLEDGEMENTS}

I want to give sincere thanks to Corinne Manogue at Oregon State University, John Thompson at the University of Maine, as well as my adviser Dr. Megan Wawro, for their help and contribution to this work.

This material is based upon work supported by the National Science Foundation under Grant Number DUE1452889. Any opinions, findings, and conclusions or recommendations expressed in this material are those of the author and do not necessarily reflect the views of the National Science Foundation.
[1] N. Almog, B.-S. Ilany, Educational Studies in Mathematics 81, (2012).

[2] A. Sierpinska, G. Bobos, A. Pruncut, Educational Studies in Mathematics 78, (2011).

[3] M. Zandieh, in Research in Collegiate Mathematics Education IV, edited by E. Dubinsky, A. Schoenfeld, J. Kaput (American Mathematical Society, Providence, RI, 2000).

[4] E. Lockwood, Journal of Mathematical Behavior, 32 (2013).
[5] E. von Glasersfeld, Radical Constructivism: A Way of Knowing and Learning, (Falmer Press, London, 1995).

[6] E. Lockwood, Journal of Mathematical Behavior, 32 (2013), p. 252.

[7] A. diSessa, D. Hammer, B. Sherin, T. Kolpakowski, Journal of Mathematical Behavior, 10 (1991).

[8] A. diSessa, Cognition and Instruction, 22 (2004).

[9] M. Zandieh, J. Ellis, C. Rasmussen, Educational Studies in Mathematics, 95 (2017) 